\title{
Les eaux carbo-gazeuses et minéralisées du sud-est de la Belgique
}

\author{
A. Monjoie \\ Professeur à l'Université de Liège
}

\begin{abstract}
Dans le sud de la Belgique se situent les principales sources d'eaux minérales et carbo-gazeuses de la Belgique. Leurs caractéristiques résultent de l'histoire géologique particulière de cette région.
\end{abstract}

\section{D CADRE GÉOLOGIQUE GÉNÉRAL}

La géologie du sud de la Belgique est caractérisée par deux grandes entités structurales : le synclinorium de Namur au nord, le synclinorium de Dinant au sud, ce deuxième étant charrié sur le premier par le jeu de la faille eifelienne (fig. 1). L'un et l'autre sont constitués à la base par des formations de phyllades, quartzophyllades et quartzites du Cambro-Silurien surmontées par des dépôts terrigènes et carbonatés transgressifs du Dévonien et du Carbonifère. Cette structure remonte dans le sud-est de la Belgique, soit en raison des ennoyages vers l'ouest du Synclinorium de Dinant, soit, ce qui est plus probable, par le jeu d'accidents subméridiens. Il en résulte que, dans cette zone, le massif de Stavelot, prolongement du Synclinorium de Dinant, est constitué presque uniquement par les formations cambrosiluriennes et éodévoniennes.

Pour les mêmes motifs, la faille eifelienne y serait moins profonde, avec l'énigmatique "fenêtre de Theux " qui laisse apparaître les formations dévoniennes et carbonifères du synclinorium de Namur sous-jacent.
La région a été affectée par les plissements calédoniens et hercyniens et par le jeu de nombreuses failles transverses subméridiennes.

Après l'orogénèse calédonienne affectant les formations cambro-siluriennes il y a 500 millions d'années, les reliefs existants ont été progressivement aplanis, altérés, puis recouverts par les formations du Dévonien et du Carbonifère.

L'orogenèse hercynienne a ensuite édifié une importante chaîne de montagnes qui, à son tour, a été érodée et pénéplanée il y a environ 250 millions d'années.

Cette pénéplaine, dont les restes correspondent au sommet des crêtes ardennaises, dont celle de la Grande Vecquée au sud de Spa, fut soumise à une intense altération, pour être ensuite recouverte au Secondaire par les formations de craies à silex du Crétacé. Celles-ci, solubles, se sont dissoutes rapidement, laissant subsister les matériaux insolubles, formant ainsi des lambeaux d'argile à silex. Au Tertiaire, de nouveaux dépôts marins constitués de sables ont recouvert la région. Au Quaternaire, le climat glaciaire, puis la fonte des neiges et glaces ont entraîné une érosion importante de la pénéplaine et des dépôts résiduels qui la recouvrent avec encaissement des vallées. Seuls les sommets conservent des reliques de l'ancienne pénéplaine: argile à silex du Crétacé et lambeaux de sables tertiaires.

Sous cette ancienne pénéplaine, les roches du substratum sont complètement déminéralisées sur 50 à $100 \mathrm{~m}$

\section{Carbonated and mineral waters in south-east Belgium}

Carbonated water and different types of mineral waters are found in south-east Belgium.

Carbonated waters, located mainly in the areas of Spa and Chevron, are old, deep waters the mineralisation of which is largely a function of the lithology of the rocks they pass through. The gas, mainly $\mathrm{CO}_{2}$, appears to come from deep sedimentary limestone rocks.

The mineral waters in the Spa region have low mineralisation. The phenomenon is related to post-primary prehistoric changes.

The waters at Chaudfontaine are characteristic of limestone formations but have temperatures higher than normal. 


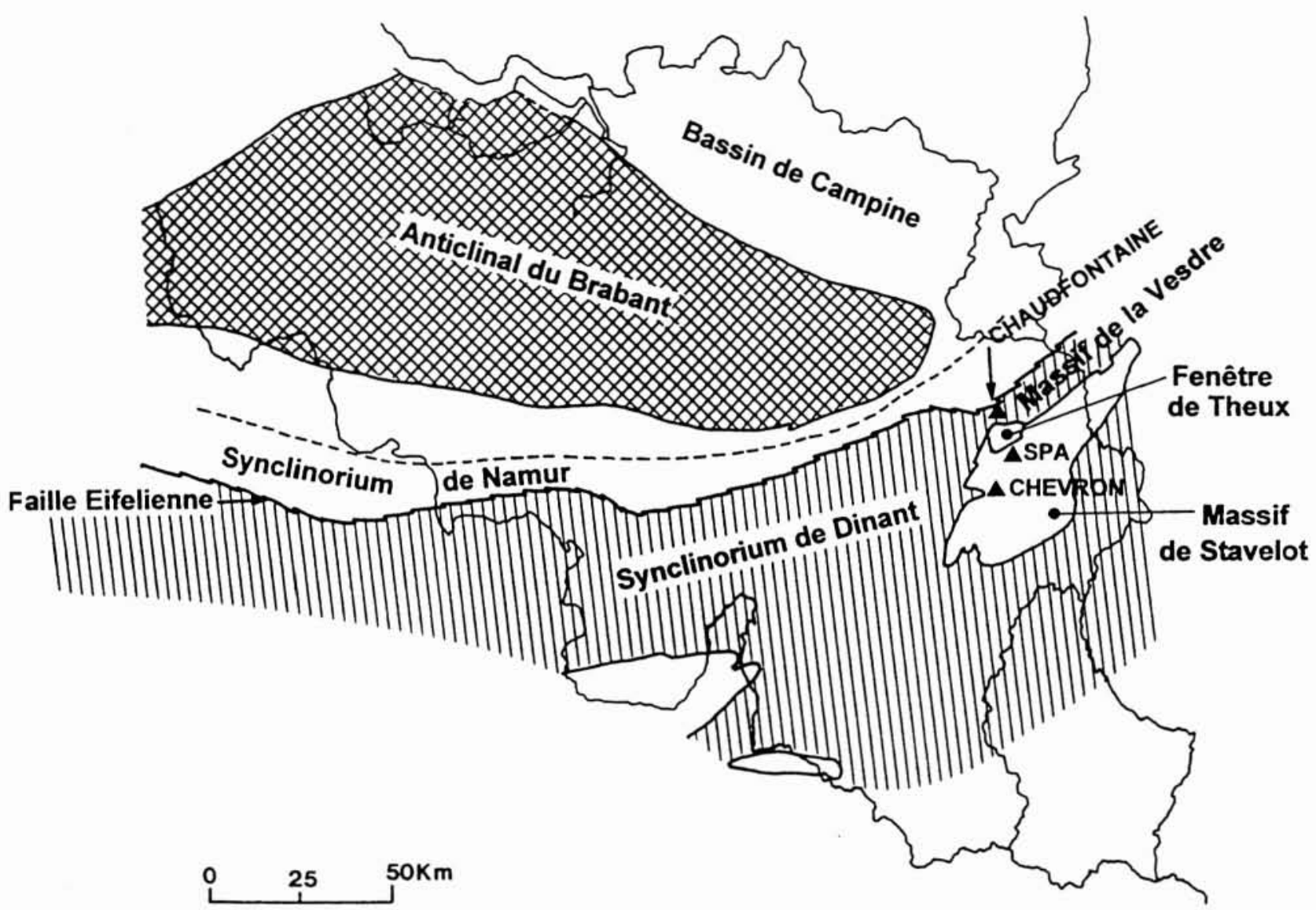

1. Carte tectonique sommaire du socle paléozö̈que de la Belgique (d'après Prodrome de la description géologique de la Belgique).

d'épaisseur suite à la paléo-altération post-hercynienne et peut-être à la paléo-altération post-calédonienne et antédévonienne.

C'est dans ce contexte géologique particulier qu'émergent les principales eaux minérales et carbo-gazeuses de la Belgique dont les plus caractéristiques sont :

- les eaux carbo-gazeuses des régions de Spa et de Bru-Chevron,

- les eaux pauci-minéralisées de la région de Spa,

- les eaux thermales de Chaudfontaine.

\section{LES EAUX CARBO-GAZEUSES DES RÉGIONS DE SPA ET DE BRU- CHEVRON}

Le schéma hydrogéologique des venues d'eaux minérales carbo-gazeuses correspond à une remontée de l'eau du substratum profond par le biais d'accidents transverses aquifères. Près de la surface, il y a dispersion de l'eau dans les fissures des zones détendues et altérées et une même venue d'eau profonde donne généralement lieu à plusieurs exutoires ou pouhons.

L'eau carbo-gazeuse reste souvent piégée sous les nappes aquifères superficielles et ne sourd en surface qu'aux endroits de couverture moins épaisse ou de plus forte perméabilité des terrains.
Les pouhons semblent localisés sur le tracé d'accidents orientés N-S, souvent à la rencontre avec d'autres accidents orientés NNE-SSW ou NW-SE. L'eau est d'origine météorique ancienne. Les datations au tritium donnent des valeurs de 1 à 5 uT. Cette eau infiltrée à grande profondeur s'est, au contact des roches, chargée en gaz carbonique et en sels minéraux. Dans les zones failleuses, le $\mathrm{CO}_{2}$, en se dégageant, permet le jaillissement rapide des eaux avec conservation de leurs caractéristiques acquises lors de leur lente migration en profondeur. Le dégagement de gaz ne pouvant avoir lieu que si la contre-pression en surface est faible, les pouhons sont localisés dans les vallées ou en altitude limitée, rarement supérieure à $440 \mathrm{~m}$ dans la région de Spa. Plus haut, la contre-pression due à la nappe superficielle est suffisamment élevée pour empêcher le dégagement de gaz.

L'origine du $\mathrm{CO}_{2}$ et les causes de la minéralisation des eaux ont fait l'objet d'une étude particulière dans la région de Bru-Chevron (R. Wollast et al., 1985 et 1987).

Le contexte géologique est semblable à celui de Spa mais on se situe en bordure du contact entre le massif de Stavelot et le bord oriental du Synclinorium de Dinant. Le secteur est affecté par une importante faille de charriage : la faille de Xhoris, et par plusieurs accidents transverses sub-méridiens et est-ouest. La composition chimique des eaux et leur teneur en ${ }^{13} \mathrm{C}$ semble exclure l'hypothèse de $\mathrm{CO}_{2}$ juvénile ou provenant de la décomposition de matières organiques. Une origine résultant de la dissolution de 
calcaires marins au départ d'un acide fort semble la plus vraisemblable, l'acide pouvant résulter d'une altération de pyrites en profondeur. Les hautes teneurs en silice et en magnésie des eaux militent pour l'existence d'une attaque des silicates en plus de celle des carbonates. Les remontées d'eaux carbo-gazeuses se font, comme à Spa, dans les plans de failles, avec concentration à la recoupe d'accidents plus ou moins orthogonaux. Parfois situés à proximité les uns des autres, les pouhons peuvent présenter des variations chimiques significatives en raison de cheminements différents dans les terrains supérieurs.

Dans la région de Spa, on peut distinguer trois ensembles se différenciant légèrement au point de vue chimique.

\section{a. Pouhons du centre ville (Pierre Le Grand - Condé - Armes d'Autriche)}

Leur altitude est de plus ou moins $250 \mathrm{~m}$. Ils sont les plus minéralisés, avec des teneurs en Ca et en $\mathrm{Mg}$ supérieures à $20 \mathrm{mg} / \mathrm{l}$ et une conductivité supérieure à $400 \mu \mathrm{S} / \mathrm{cm}$. La teneur en fer varie entre 10 et $20 \mathrm{mg} / \mathrm{l}$. Le $\mathrm{CO}_{2}$ libre se situe entre 1600 et $3000 \mathrm{mg} / \mathrm{l}$. Le $\mathrm{SiO}_{2}$ est de l'ordre de $50 \mathrm{mg} / \mathrm{l}$. Les teneurs en $\mathrm{K}(>3 \mathrm{mg} / \mathrm{l})$ et $\mathrm{Na}(>30 \mathrm{mg} / \mathrm{l})$ sont également élevées.

\section{b. Pouhons « de Nivezé » (Marie Henriette - Welling- ton - Tonnelet)}

Leur altitude est comprise entre 305 et $320 \mathrm{~m}$. Par rapport aux pouhons du centre ville, ils sont moins minéralisés : $\mathrm{Ca} \quad(<12 \mathrm{mg} / \mathrm{l}), \quad \mathrm{Mg} \quad(<8 \mathrm{mg} / \mathrm{l}), \quad \mathrm{Na} \quad(<18 \mathrm{mg} / \mathrm{l})$, $\mathrm{K}(<2 \mathrm{mg} / \mathrm{l}), \mathrm{SiO}_{2}(<30 \mathrm{mg} / \mathrm{l})$.

La conductivité est proche de $200 \mu \mathrm{S} / \mathrm{cm}$. Les teneurs en $\mathrm{Fe}$ et en $\mathrm{CO}_{2}$ libre restent du même ordre $(\mathrm{Fe}: 14$ à $18 \mathrm{mg} / \mathrm{l} ; \mathrm{CO}_{2}$ libre : variant de 2000 à $4000 \mathrm{mg} / \mathrm{l}$ ).

L'eau de Marie Henriette, commercialisée, présente les concentrations indiquées sur le tableau 1.

\section{Tableau 1.}

\begin{tabular}{|c|c|c|c|c|c|c|c|c|c|c|}
\hline $\begin{array}{l}\mathrm{Na} \\
\mathrm{mg} / 1\end{array}$ & $\begin{array}{c}\mathrm{K} \\
\mathrm{mg} / \mathrm{l}\end{array}$ & $\begin{array}{c}\mathrm{Ca} \\
\mathrm{mg} / 1\end{array}$ & $\begin{array}{c}\mathrm{Mg} \\
\mathrm{mg} / 1\end{array}$ & $\begin{array}{c}\mathrm{Cl} \\
\mathrm{mg} / \mathrm{l}\end{array}$ & $\begin{array}{l}\mathrm{SO}_{4} \\
\mathrm{mg} / 1\end{array}$ & $\begin{array}{l}\mathrm{NO}_{3} \\
\mathrm{mg} / \mathrm{l}\end{array}$ & $\begin{array}{c}\mathrm{HCO}_{3} \\
\mathrm{mg} /\end{array}$ & $\begin{array}{l}\mathrm{SiO}_{2} \\
\mathrm{mg} / 1\end{array}$ & $\begin{array}{c}\text { Résidu } \\
\text { sec } \\
\mathrm{mg} / 1\end{array}$ & $\begin{array}{c}\mathrm{CO}_{2} \\
\text { naturel } \\
\text { gr/litre }\end{array}$ \\
\hline 10,5 & 1,3 & 11 & 7 & 9,5 & 6,5 & 0,5 & 75 & 15 & 95 & 3,8 \\
\hline
\end{tabular}

c. Pouhons de la route des Fontaines: Sauvenière Groesbeck - Géronstère - Hornay - Barisart - Pia Delcor

Tous sont situés à une altitude proche de $440 \mathrm{~m}$, sauf Barisart. Par rapport aux autres pouhons, ils ont une composition intermédiaire entre ceux du centre ville et ceux de « Nivezé ».

Cependant, on constate que :

- Sauvenière et Groesbeck ont des teneurs en Ca semblables aux pouhons du centre ville, et plus élevées en $\mathrm{Fe}$, - la teneur en $\mathrm{SiO}_{2}$ est plus faible (environ $10 \mathrm{mg} / \mathrm{l}$ ), sauf au pouhon de Barisart,

- la teneur en $\mathrm{CO}_{2}$ libre est la plus faible pour Géronstère et Hornay.
A Bru-Chevron, la chimie des eaux varie selon les émergences.

La composition de l'eau commercialisée Bru-Chevron est indiquée sur le tableau 2.

Tableau 2.

\begin{tabular}{|c|c|c|c|c|c|c|c|c|c|c|}
\hline $\mathrm{Na}$ & $\mathrm{K}$ & $\mathrm{Ca}$ & $\mathrm{Mg}$ & $\mathrm{Cl}$ & $\mathrm{SO}_{4}$ & $\mathrm{NO}_{3}$ & $\mathrm{HCO}_{3}$ & $\mathrm{SiO}_{2}$ & $\begin{array}{c}\text { Résidu } \\
\text { sec } \\
\mathrm{mg} / 1\end{array}$ & $\begin{array}{c}\mathrm{CO}_{2} \\
\text { ng/urel } \\
\mathrm{m} / / \text { litre }\end{array}$ \\
\hline 10 & 1,5 & 23 & 22 & 4 & 5 & 0,7 & 209 & 17 & 160 & 4,4 \\
\hline
\end{tabular}

Il convient de noter les teneurs pratiquement égales en calcium et en magnésium et des teneurs en silice élevées. D'autres émergences présentent soit des valeurs en calcium beaucoup plus élevées, pouvant être de 40 à $90 \mathrm{mg} / \mathrm{l}$, avec des teneurs en magnésium de 18 à $30 \mathrm{mg} / \mathrm{l}$, nettement inférieures à celles du calcium, et des teneurs en silice pratiquement équivalentes. D'autres gardent les mêmes teneurs en calcium (de l'ordre de 20 à $30 \mathrm{mg} / \mathrm{l}$ ) mais avec des teneurs en magnésium plus faibles (de 15 à $16 \mathrm{mg} / \mathrm{l}$ ) et des teneurs en silice de 20 à $30 \mathrm{mg} / \mathrm{l}$.

Ceci témoigne de la recoupe de terrains différents par les eaux carbo-gazeuses en phase de remontée et/ou des circulations à vitesse plus grande, entraînant des modifications de chimisme significatives.

\section{III — LES EAUX PAUCI-MINÉRALISÉES}

La plus renommée est l'eau de Spa Reine. Ce type d'eau résulte de la paléoaltération intense qui a affecté les sommets au sud de Spa (crête de la Grande Vecquée - cote $560-570 \mathrm{~m}$ ), résultat des pénéplanations post-calédonienne et post-hercynienne (fig. 2 et 3 ).

Les eaux qui s'infiltrent dans cette zone ne rencontrent plus que très peu d'ions solubilisables, donnant des eaux très faiblement minéralisées, eaux que l'on retrouve depuis les sommets jusqu'à la cote d'environ $440 \mathrm{~m}$.

En contrebas, le substratum a été moins affecté par la paléoaltération et les eaux sont légèrement plus minéralisées (type Barisart) mais restent peu minéralisées car les roches (phyllades, schistes et quartzites) sont peu solubles.

Les compositions moyennes en éléments majeurs de ces deux types d'eau sont données dans le tableau 3.

Tableau 3.

\begin{tabular}{|c|c|c|c|c|c|c|c|c|c|c|c|}
\hline & $\begin{array}{l}\mathrm{Na} \\
\mathrm{mg} / \mathrm{l}\end{array}$ & $\begin{array}{c}\mathrm{K} \\
\mathrm{mg} / \mathrm{l}\end{array}$ & $\begin{array}{l}\mathrm{Ca} \\
\mathrm{mg} /\end{array}$ & $\begin{array}{l}\mathrm{Mg} \\
\mathrm{mg} / \mathrm{l}\end{array}$ & $\mathrm{Cl}$ & $\begin{array}{l}\mathrm{SO}_{4} \\
\mathrm{mg} / 1\end{array}$ & $\begin{array}{l}\mathrm{NO}_{3} \\
\mathrm{mg} / \mathrm{l}\end{array}$ & $\begin{array}{c}\mathrm{HCO}_{3} \\
\mathrm{mg} / 1\end{array}$ & $\begin{array}{l}\mathrm{SiO}_{2} \\
\mathrm{mg} / 1\end{array}$ & $\begin{array}{c}\text { Résidu } \\
\text { sec } \\
\mathrm{mg} / 1\end{array}$ & $\begin{array}{l}\mathrm{CO}_{2} \\
\text { naturel } \\
\text { gr/litre }\end{array}$ \\
\hline $\begin{array}{l}\text { Spa } \\
\text { Reine }\end{array}$ & 3 & 0,5 & 3,5 & 1,3 & 5 & 6,5 & 1,9 & 11 & 7 & 33 & - \\
\hline $\begin{array}{l}\text { Spa } \\
\text { Barisart }\end{array}$ & 5 & 0,5 & 5,5 & 1,5 & 5,5 & 7,5 & 1,5 & 18 & 10 & 49 & - \\
\hline
\end{tabular}






2. Schéma géologique d'après les cartes géologiques du service géologique de Belgique et les travaux des L.G.I.H. (U.L.G.).

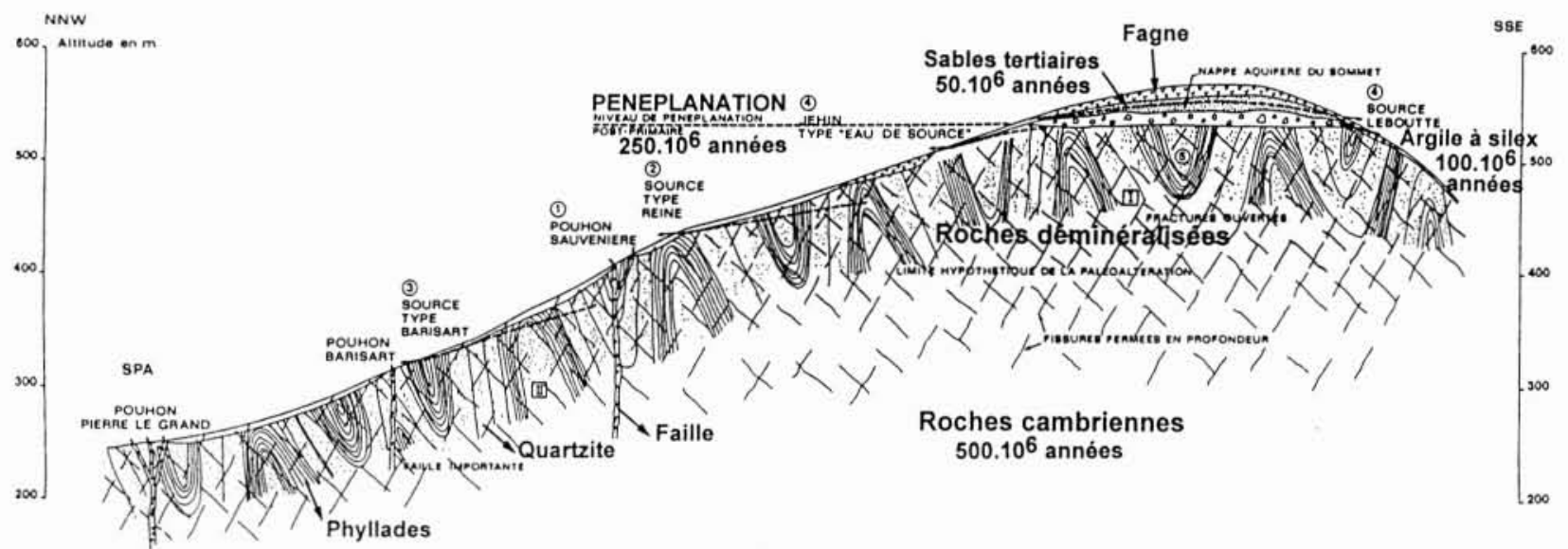

\section{Région de Spa. Coupe géologique.}

Les datations tritium indiquent un âge très récent de ces eaux, de l'ordre de 6 mois à quelques années.

Les nappes aquifères correspondantes sont logées dans les fissures et failles des quartzites, phénomènes abondants en raison des deux plissements calédonien et hercynien ayant affecté la région. Par contre, les schistes et phyllades se broyant en argile. ils sont imperméables et compartimentent les nappes aquiferes. Les contacts quartzitesschistes et les zones faillées constituent ainsi des sites préférentiels de captage. La prospection de ces eaux implique la localisation des zones quartzitiques fissurées.

Les méthodes utilisées dans ce but sont l'électriquerésistivité et la sismique-réfraction, complétées par la prospection magnétique et électromagnétique.

Les zones d'extension de ces deux types d'eau sont donc fonction de la topographie sous la cote de l'ancienne pénéplanation, superficies limitées impliquant une gestion stricte, fonction du cycle de l'eau et du volume des réserves des gisements aquiferes.

La très faible minéralisation de ces eaux impose des contraintes importantes dans les périmètres de protection, tant au niveau des activités humaines que du maintien du couvert végétal constitué principalement de feuillus, épicéas et tourbières.

D'autre part, la présence d'eaux carbo-gazeuses en dessous d'un gisement d'eau très peu minéralisée pose un important problème au niveau de la gestion de ces deux potentiels. Si par pompage, on rabat de façon notable la nappe supérieure, on réduit la surpression qu'elle exerce et on risque de fortes arrivées d'eaux chargées en $\mathrm{Fe}$ et en gaz carbonique, compromettant toute la valeur de la nappe superficielle. D'autre part, si l'on pompe de façon trop 
importante les eaux carbo-gazeuses, on entraîne une dilution de celles-ci par les eaux superficielles, avec modification du chimisme. Ceci implique une étude complète du bilan des aquifères et une gestion des périmètres de protection de façon à éviter tout déséquilibre dans le milieu hydrologique.

\section{LES EAUX THERMALES DE CHAUD- FONTAINE}

Elles ont été étudiées en détail par J.M. Graulich (1983). La région de Chaudfontaine est caractérisée par des venues d'eau présentant des températures de 30 à $40^{\circ} \mathrm{C}$, avec des gradients de l'ordre de $1{ }^{\circ} \mathrm{C}$ par $10 \mathrm{~m}$ dans la zone géothermique, contre $1{ }^{\circ} \mathrm{C}$ par 30 à $100 \mathrm{~m}$ dans les formations environnantes.

Il s'agit d'eaux bicarbonatées calciques dont la composition chimique est donnée dans le tableau 4.
Tableau 4.

\begin{tabular}{|c|c|c|c|c|c|c|c|c|c|}
\hline $\begin{array}{c}\mathrm{Na} \\
\mathrm{mg} /\end{array}$ & $\begin{array}{c}\mathrm{K} \\
\mathrm{mg} /\end{array}$ & $\begin{array}{c}\mathrm{Ca} \\
\mathrm{mg} / 1\end{array}$ & $\begin{array}{c}\mathrm{Mg} \\
\mathrm{mg} / 1\end{array}$ & $\begin{array}{c}\mathrm{Cl} \\
\mathrm{mg} / 1\end{array}$ & $\begin{array}{c}\mathrm{SO}_{4} \\
\mathrm{mg} /\end{array}$ & $\begin{array}{c}\mathrm{NO}_{3} \\
\mathrm{mg} / 1\end{array}$ & $\begin{array}{c}\mathrm{HCO}_{3} \\
\mathrm{mg} /\end{array}$ & $\begin{array}{c}\mathrm{SiO}_{2} \\
\mathrm{mg} / 1\end{array}$ & $\begin{array}{c}\text { Résidu } \\
\text { sec } \\
\mathrm{mg} / 1\end{array}$ \\
\hline 44 & 2,5 & 65 & 18 & 35 & 40 & 22 & 305 & 14 & 385 \\
\hline
\end{tabular}

Ces venues sont localisées dans des aquifères de calcaires frasniens affectés par un pli anticlinal et situé dans le massif charrié au-dessus de la faille eifelienne. Celle-ci affleure quelques kilomètres au nord et se trouve à 300 à $400 \mathrm{~m}$ de profondeur dans la zone thermale (fig. 4).

L'origine des eaux semble météorique sur la base des teneurs en deutérium et en $\mathrm{O}^{18}$. La datation au tritium inférieure à 1,2 unité confirme un âge de plus de 30 années. Les venues en surface subissent une certaine dilution par

\section{$\mathbf{N}$}

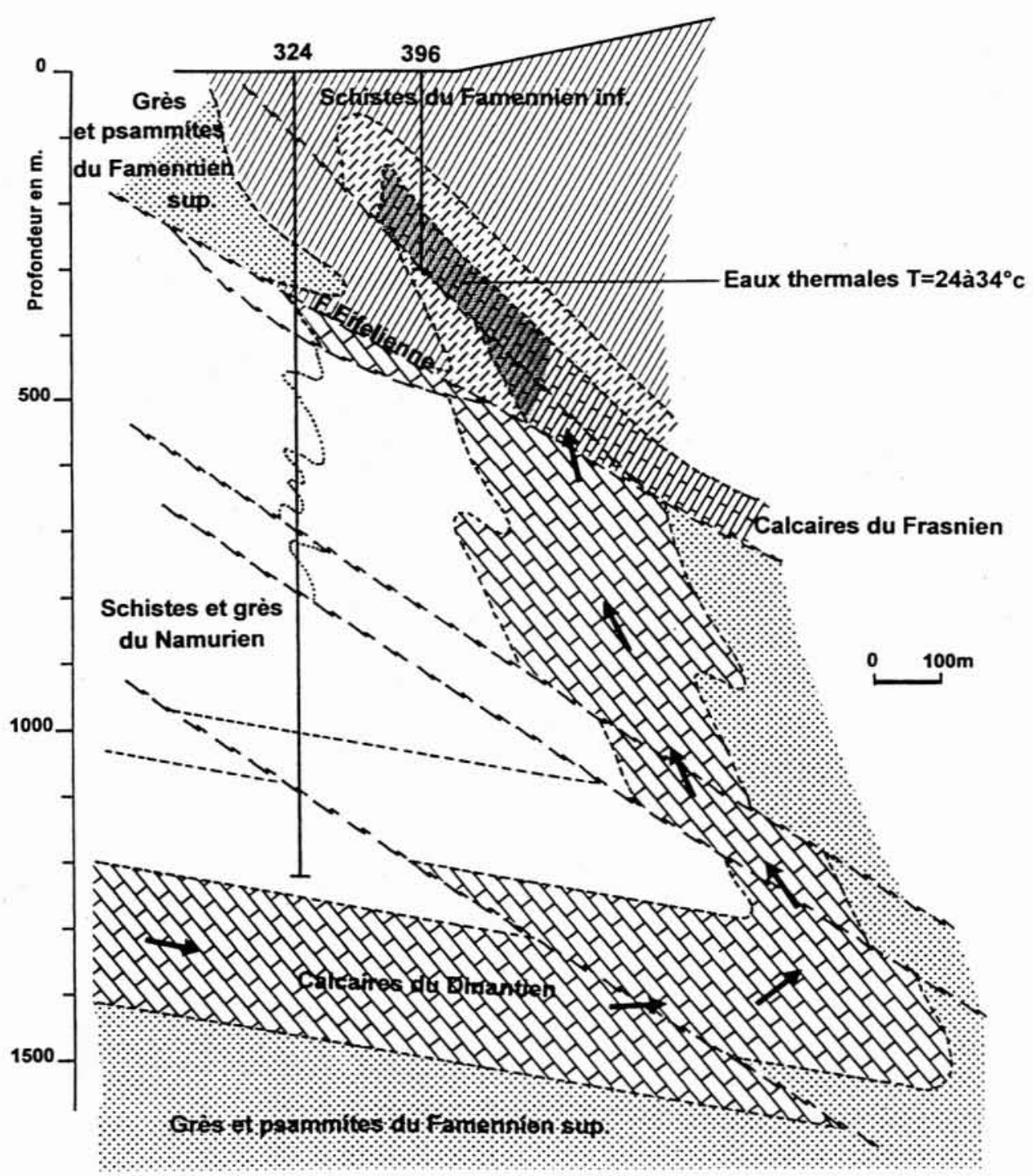

4. Chaudfontaine. Coupe géologique passant par les sondages 324 et 396 (d'après J.M. Graulich, 1983). 
des eaux récentes entraînant des baisses de température jusqu'à $30^{\circ} \mathrm{C}$ et une datation au tritium plus jeune (10 à 12 uT). Le géothermomètre, basé sur la teneur en quartz, indique une température de l'ordre de $50{ }^{\circ} \mathrm{C}$, ce qui implique, compte tenu des gradients thermiques de la région, que les eaux pénètrent à plus de $1500 \mathrm{~m}$ de profondeur.

Ceci peut être envisagé si, par le jeu de la faille eifelienne, le gisement géothermal actuel est mis en contact avec des calcaires du Carbonifère du synclinorium de Namur sous-jacent à la faille eifelienne.

\section{Bibliographie}

CENTRE SPA D'INFORMATION DE L'EAU (1993). — L'eau et la santé, pp. 20-30.

DEFOSSE C. et WERY A. (1975). - Recherche sur les pouhons des Ardennes liégeoises, Revue du Centre Belge de Documentation des Eaux (CEBEDOC), 375, pp. 1-7.

FOURMARIER P. (1955). - Réflexions au sujet de l'origine des eaux thermales de Chaudfontaine, Bulletin de la Société Géologique de Belgique, T. 78, pp. 491-510.
Graulich J.M. (1983). - L'hydrogéologie thermale de Chaudfontaine, Bulletin de la Société Géologique de Belgique, T. 92, fasc. 3, pp. 195-212.

MONJOIE A. et CAJOT O. - Etude des eaux minérales de la région de Spa, Rapports pour la Société Spa Monopole, de 1975 à 1994.

Monjoie A., Cajot O. et Calembert L. (1978). - Etude hydrogéologique du site de Bru. Etude pour la Compagnie Générale de Chevron.

Monjoie A., CAjot O. et CAlembert L. (1979). - Reconnaissance du pouhon Sart comme eau minérale, Rapport à la Compagnie Générale de Chevron.

VAN BENEDEN G. (1947). - Recherches sur l'origine et la genèse des eaux minérales de Spa et des Ardennes, Journal de Pharmacie de Belgique, pp. 1-26 et 60-77.

Wollast R. et al. (1985). - Etude du périmètre de protection de la zone de Stoumont et environs, Ministère de la Région Wallonne pour l'eau, Rapport final.

Wollast R. et Wollast A. (1987), - Etude géochimique des eaux carbo-gazeuses de la région de Stoumont, Colloque sur « Les eaux souterraines en Wallonie », Ministère de la Région Wallonone, Bilan et Perspectives Eso 87, pp. 152167. 